\title{
MODELACION DE PUENTE PEATONAL EN ACERO PARA LA MEJORA DE LA MOVILIDAD PEATONAL DEL SECTOR DE LA AVENIDA 68 ENTRE CALLES 63 Y 64
}

\author{
Hernández Salazar Sebastián Camilo \& Ramos Arenas Andrés Felipe \\ Mentor académico. Julio Oswaldo Torres, Magister en Estructuras \\ Estudiante de Ingeniería civil, Universidad La Gran Colombia, Colombia, hernandezsebastiancamilo@gmail.com \\ Estudiante de Ingeniería civil, Universidad La Gran Colombia, Colombia, andresfelipe.ramos@ulagrancolombia.edu.co
}

\begin{abstract}
Resumen: El puente peatonal ubicado en la Avenida Carrera 68 entre las calles 63 y 64 en la localidad de Engativá, Bogotá, es utilizado por numerosas personas que transitan en este sector, estos desarrollan actividades deportivas y recreativas debido a que allí se encuentran diferentes instituciones y ambientes públicos. El puente peatonal se usa para cruzar la Avenida 68, esta avenida tiene una alta probabilidad de accidentalidad debido a su alto flujo vehicular, de acuerdo a lo mencionado anteriormente, la alternativa más viable es la utilización del puente, mas sin embargo, por el alto flujo de personas y el tiempo de construido ,este presenta daños estructurales, generando una percepción de inseguridad al utilizarlo, por ello, se cree necesario el diseño de un nuevo puente peatonal que cumpla con la normativa vigente, la Norma Colombiana de Diseño de Puentes - LRFD - CCP 14 [1] la cual trata del diseño y análisis estructural de los puentes que se realizan en Colombia. Se realizó el diseño y la modelación de un nuevo puente peatonal mediante el siguiente proceso: descripción cualitativa de la estructura (mediciones de campo), análisis dinámico causado por el sismo y chequeo de derivas. La modelación se realizó por medio del software SAP2000; luego de realizar la modelación se obtuvieron desplazamientos que no exceden los desplazamientos máximos permitidos que corresponden al $1 \%$ de la altura total del puente, por otra parte, la capacidad de carga de los elementos usados en el diseño no excede el $80 \%$.
\end{abstract}

Palabras claves: Derivas, evaluación estructural, mediciones de campo, modelación, movilidad peatonal, peatón, Puente peatonal.

\begin{abstract}
The pedestrian bridge located on Avenue Career 68 between streets 63 and 64 in the location of Engativá, Bogota, is used by many people passing in this sector; they develop sports and recreational activities because there are different institutions and environments public. The pedestrian bridge used to cross 68th Avenue, this avenue has a high probability of accidents due to high traffic flow, according to the above, the most viable alternative is the use of the bridge, but nevertheless, the high flow of people and time built, this structural damage, creating a perception of insecurity to use, therefore, the design of a new pedestrian bridge that complies with current regulations deemed necessary, the Colombian Standard bridge design - LRFD - CCP 14 [1] which discusses the design and structural analysis of bridges that are made in Colombia. The design and modeling of a new footbridge was performed by the following process: qualitative description of the structure (field measurements), dynamic analysis caused by the earthquake and check drift. The modeling was performed using the software SAP2000; after performing the modeling displacement not
\end{abstract}

exceeding the maximum permissible displacements corresponding to $1 \%$ of the total height of the bridge, on the other hand, the capacity of the elements used in the design does not exceed $80 \%$ were obtained.

Key words: Drifts, field measurements, footbridge, modeling, pedestrian mobility, pedestrian, structural assessment.

\section{INTRODUCCIÓN}

Esta investigación se realizó con el objeto de identificar una problemática de la comunidad y dar una solución desde el punto de vista Ingenieril.

La estructura del puente peatonal ubicado en la Avenida 68 entre calles 63 y 64 se encuentra en un estado de deterioro; motivo por el cual los transeúntes del sector tienen una percepción de inseguridad pero aun así lo utilizan porque es la alternativa más segura para cruzar la Avenida 68. En la estructura se pueden observar fisuras en las conexiones Columna-Viga y en la mitad del tablero se encuentra un agujero que parece ser del diseño estructural pero para el ojo inexperto parece una falla en el puente lo cual genera inseguridad en las personas que utilizan este puente.

El objetivo general de este proceso es proponer una posible mejora en la movilidad peatonal del puente peatonal ubicado en la Avenida 68 entre las calles 63 y 64 desde el punto de vista ingenieril.

Para este estudió se tomó la normativa vigente [1] y se realizaron pruebas de campo con el fin de identificar las condiciones actuales de la estructura y de esta forma iniciar la respectiva modelación de un nuevo diseño en el software SAP2000, y a través de éste observar la capacidad de carga de los elementos estructurales y las derivas obtenidas las cuales deben corresponder al $1 \%$ de la altura total del puente.

A continuación se presenta el procedimiento detallado del diseño y modelación del nuevo puente, en el cual se indica los métodos y ensayos en campo realizados para obtención de los datos, con el propósito de realizar la modelación correspondiente en el software mencionado anteriormente. Por último, se plantea la alternativa para la mejora de la movilidad peatonal. 


\section{METODOLOGÍA}

\section{A. Estudios realizados en la zona}

En la zona de estudio se realizaron las siguientes actividades:

- Registro fotográfico.

- Toma de medidas del puente peatonal.

B. Delimitación de la zona de estudio (localización).

El área geográfica de la investigación se ubica en el departamento de Cundinamarca en la ciudad de Bogotá D.C. En la localidad de Engativá en la dirección Avenida carrera 68 No. 64-45 se ubica el puente que actualmente está construido (Ilustración 1).

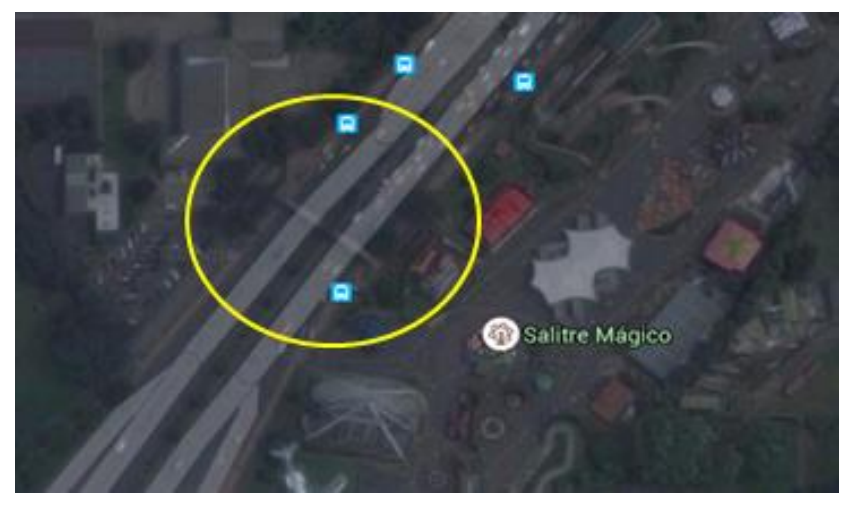

Ilustración 1. Localización de puente peatonal.

\section{Condiciones del uso del suelo.}

Este sector tiene un uso de suelo dotacional que se refiere a que tiene equipamiento colectivo donde ubicamos la parte de la educación y también tiene un equipamiento deportivo y recreativos donde incluye parques públicos.

En el sector de la Avenida carrera 68 No. 64 - 45 se encuentra el colegio Cafam, donde los estudiantes toman el puente para poder desplazarse para el colegio o a sus casas.

\section{HERRAMIENTAS DE ANÁLISIS.}

El análisis del nuevo modelo del puente peatonal se realizó teniendo como referencia la Norma Colombiana de Diseño de Puentes - LRFD - CCP 14 dentro de la cual se especifica [1]:

$\checkmark \quad$ Las cargas permanentes y variantes que el puente soportara a lo largo de su vida útil.

$\checkmark \quad$ Las diferentes combinaciones de carga que se pueden presentar en un ambiente realista.

$\checkmark$ El espectro sísmico que se debe tomar para desarrollar el análisis símico de la zona de investigación.
Para proceder a la realización del nuevo modelo se analizó con anterioridad el puente existente con el objeto de corroborar si este cumple o no con la demanda de peatones que transitan por él, por lo tanto, se procedió a analizar la estructura de la siguiente manera:

$\checkmark \quad$ Registro fotográfico del estado del puente existente.

$\checkmark$ Mediciones de campo.

$\checkmark$ Realizar aforos peatonales para determinar la cantidad de peatones que transitan por el puente.

$\checkmark$ Determinar el ancho mínimo de la loza por medio de la ecuación de ancho efectivo.

$\checkmark$ Realizar el diseño y la modelación del nuevo puente.

A. Registro fotográfico.

Se realizó un registro fotográfico del estado en que se encuentra el puente. A continuación se presentan las siguientes fotografías.

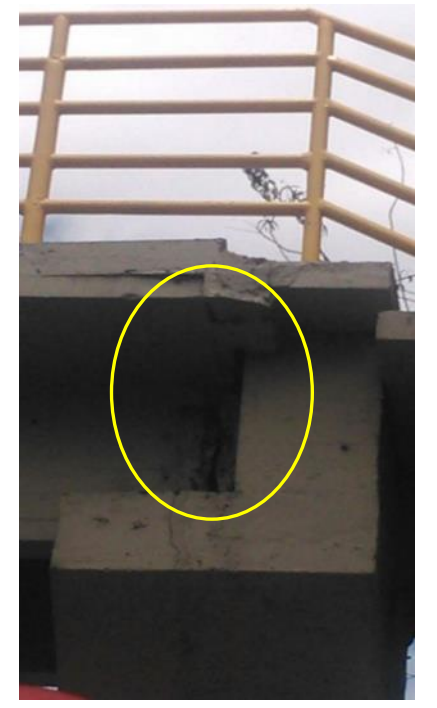

Ilustración 2.Grieta en el concreto estructural del puente peatonal en estudio.

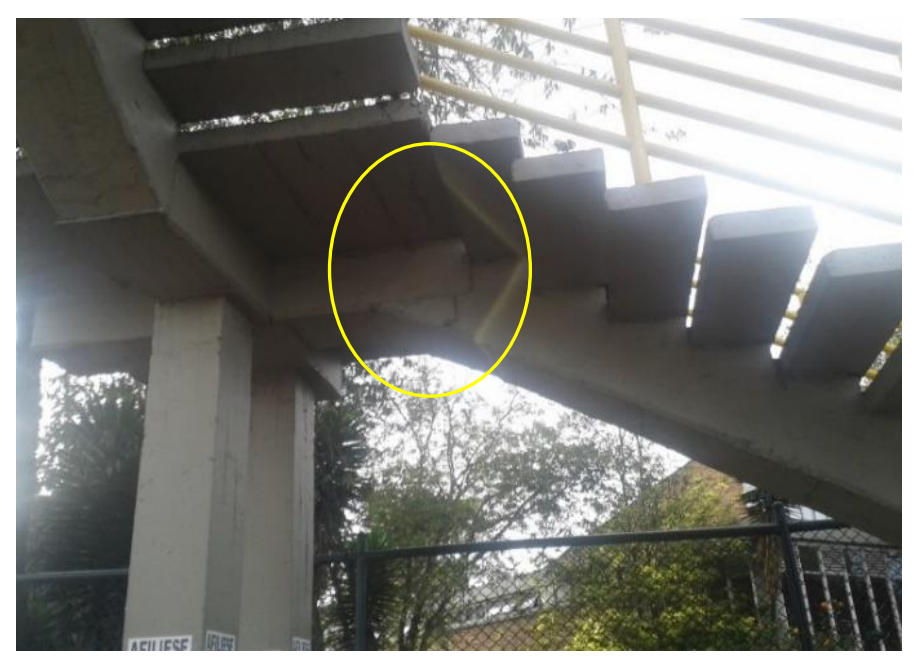

Ilustración 3.Grietas en conexiones de escaleras.

14 ${ }^{\text {th }}$ LACCEI International Multi-Conference for Engineering, Education, and Technology: "Engineering Innovations for Global Sustainability", 20-22 July 2016, San José, Costa Rica. 


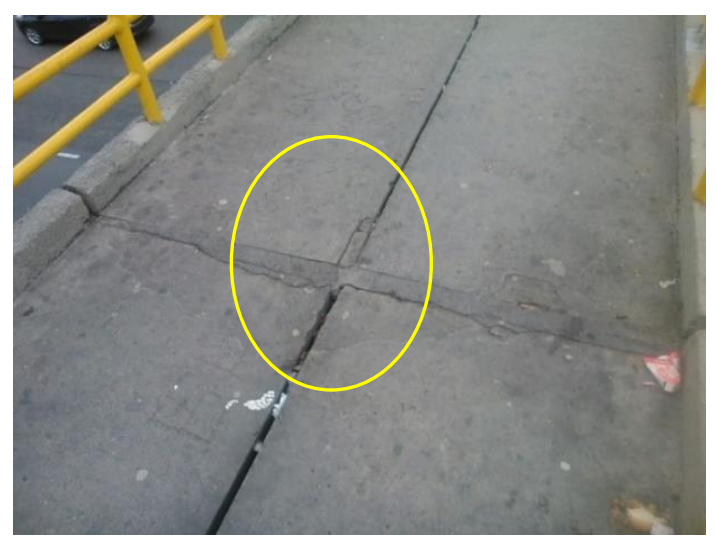

Ilustración 4. Deterioro en la losa de concreto.

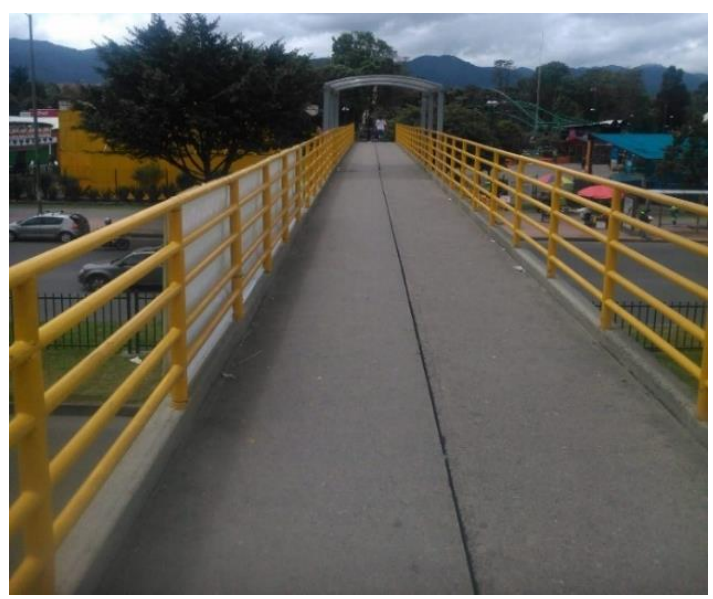

Ilustración 5. Losa puente peatonal en estudio.

B. Mediciones de campo

Las mediciones que se realizaron son:

$\checkmark \quad$ El ancho de la loza.

$\checkmark$ Las secciones de las columnas.

$\checkmark \quad$ Las secciones de las vigas.

$\checkmark$ El largo del puente.

$\checkmark \quad$ La altura del puente.

$\checkmark \quad$ Espesor de la loza.

TABLA I.

PERFILES MEDIDOS.

\begin{tabular}{cc}
\hline & PERFILES UTILIZADOS \\
\hline Loza de concreto & $\begin{array}{c}\text { Grosor de } 0.12 \mathrm{~m} \text {, ancho de la loza de } 2.33 \mathrm{~m} \mathrm{y} \\
\text { longitud de la loza de } 38.54 \mathrm{~m}\end{array}$ \\
\hline Columnas & Sección rectangular de $0.53 \mathrm{~m}$ con $0.84 \mathrm{~m}$ \\
\hline Vigas & Secciones rectangulares de alto $0.7 \mathrm{~m}$ y grosor \\
de $0.30 \mathrm{~m}$
\end{tabular}

Altura del puente De la carpeta asfáltica hasta las vigas $4.5 \mathrm{~m}$
C. Aforos peatonales.

A continuación se presentan los aforos realizados del puente peatonal:

TABLA II. RESULTADOS AFORO 1

\begin{tabular}{ccc}
\multicolumn{3}{c}{ RESULTADOS AFORO 1 } \\
\hline \multicolumn{3}{c}{ VIERNES 11/09/2015 } \\
\hline $15: 00$ & $15: 15$ & PEATONES \\
\hline $15: 15$ & $15: 30$ & 136 \\
\hline $15: 30$ & $15: 45$ & 43 \\
\hline $15: 45$ & $16: 00$ & 51 \\
\hline $16: 00$ & $16: 15$ & 50 \\
\hline $16: 15$ & $16: 30$ & 45 \\
\hline $16: 30$ & $16: 45$ & 61 \\
\hline $16: 45$ & $17: 00$ & 51 \\
\hline \multicolumn{2}{c}{ Total peatones }
\end{tabular}

TABLA III.

RESULTADOS AFORO 2

\begin{tabular}{ccc}
\multicolumn{3}{c}{ RESULTADOS AFORO 2 } \\
\hline \multicolumn{3}{c}{ SABADO 12/09/2015 } \\
\hline HORA & PEATONES \\
\hline $11: 00$ & $11: 15$ & 59 \\
\hline $11: 15$ & $11: 30$ & 73 \\
\hline $11: 30$ & $11: 45$ & 56 \\
\hline $11: 45$ & $12: 00$ & 66 \\
\hline $12: 00$ & $12: 15$ & 134 \\
\hline $12: 15$ & $12: 30$ & 109 \\
\hline $12: 30$ & $12: 45$ & 60 \\
\hline $12: 45$ & $13: 00$ & 115 \\
\hline Total peatones & $\mathbf{6 7 2}$ \\
\hline
\end{tabular}

TABLA IV. RESULTADOS AFORO 3

\begin{tabular}{|c|c|c|}
\hline \multicolumn{3}{|c|}{ MIERCOLES 16/09/2015 } \\
\hline HORA & & PEATONES \\
\hline 9:00 & $9: 15$ & 144 \\
\hline $9: 15$ & 9:30 & 157 \\
\hline $9: 30$ & $9: 45$ & 164 \\
\hline $9: 45$ & $10: 00$ & 77 \\
\hline $10: 00$ & $10: 15$ & 105 \\
\hline $10: 15$ & $10: 30$ & 92 \\
\hline $10: 30$ & $10: 45$ & 105 \\
\hline $10: 45$ & 11:00 & 80 \\
\hline Total peatones & & 924 \\
\hline
\end{tabular}

$14^{\text {th }}$ LACCEI International Multi-Conference for Engineering, Education, and Technology: "Engineering Innovations for Global Sustainability”, 20-22 July 2016, San José, Costa Rica. 


\section{Ancho efectivo.}

A continuación se mostrara la ecuación para determinar el ancho mínimo que requiere el puente para la cantidad de personas que se muestran en los aforos realizados [4].

$$
A e=\left(\frac{\mathrm{Q} 15}{q * 4}\right)=\left(\frac{\mathrm{Q} 15}{\frac{\Sigma 15}{4} * 4}\right)=\left(\frac{\mathrm{Q} 15}{\Sigma 15}\right)
$$

Ecuación 1.Ancho Efectivo [4].

- $\mathrm{Q} 15=$ Flujo pico de peatones en un periodo de 15 minutos [4].

- $\mathrm{q}$ = flujo promedio de peatones $($ Peatones $/ \mathrm{min} / \mathrm{m})$ [4].

- $\quad \Sigma 15=$ Peatones que pasan en una hora [4].

- $\mathrm{Ae}=$ Ancho efectivo de la losa del puente [4].

$$
A_{e}=\left(\frac{315}{\frac{136.25}{4} * 4}\right)=\left(\frac{315}{136.25}\right)=2.31 \mathrm{~m}
$$

Ecuación 2. Resultado Ancho Efectivo [4].

Los horarios se escogieron por la hora de salida de los centros educativos y el horario de almuerzo del fin de semana para los parques públicos y poder así determinar la cantidad de personas que utilizan el puente para desplazarse.

Se observa en el resultado de la ecuación 2 [4] que el ancho efectivo de acuerdo a los aforos se debe encontrar en el orden de $2.3 \mathrm{~m}$.

El puente actual de acuerdo a la tabla I y teniendo en cuenta la resta de los bordillos que son de 0.15 [2] $\mathrm{m}$ da un resultado de un ancho libre de $2.03 \mathrm{~m}$ por esta razón el puente existente no cumple con el ancho libre para su uso.

Por otro lado de acuerdo al documento "Criterios y parámetros de diseño para los puentes peatonales" del Instituto de Desarrollo Urbano (IDU) [3] especifica que el ancho libre mínimo debe ser del orden de $2.4 \mathrm{~m}$ por lo tanto el puente existente no cumple con los parámetros requeridos para su uso.

\section{E. Modelación nuevo puente.}

De acuerdo a los datos de la geometría del puente, registros fotográficos, ubicación y flujos peatonales obtenidos, se procedió a organizar los datos con el fin de manejar un algoritmo adecuado.

Se modeló la alternativa del puente peatonal correspondiente a la problemática presentada en la estructura analizada. Una vez organizados los datos, se procedió a iniciar el algoritmo el cual consiste en lo siguiente:

- Crear la geometría en el software SAP 2000 basado en las mediciones obtenidas.

- Definir materiales y secciones a utilizar, en esta investigación se usara perfiles en acero.

- Definir tipos de cargas (muertas, vivas, viento entre otras).

- Introducir cálculos de fuerza horizontal equivalente y análisis espectral.

- Correr modelo.

- Analizar comportamiento estructural (desplazamientos, derivas, cortantes, flectores, fuerzas axiales y normales).

- Concluir modelo mediante datos proporcionados por el software SAP 2000.

\section{MODELACIÓN DEL ESCENARIO TÉCNICO.}

La modelación de la estructura se realizó con el software SAP2000 (ver Ilustración 7), por medio de este software se realizara el análisis del comportamiento de la estructura ante diferentes tipos de cargas, con el propósito de determinar si esta cumple o no con la normativa vigente [1].

La modelación consistió en la creación de la geometría de la estructura, para este caso se usó un sistema estructural compuesto por pórticos, se realizara el ingreso de cargas y se corre el sistema.

Los criterios que se evaluaran son el de derivas (no puede ser mayor al 1\%.)[1].Posteriormente se chequeara que los elementos de la estructura no sobrepasen el $100 \%$ de su esfuerzo.

A. Datos de ingreso.

Para la modelación de la estructura, es necesario ingresar los siguientes datos:

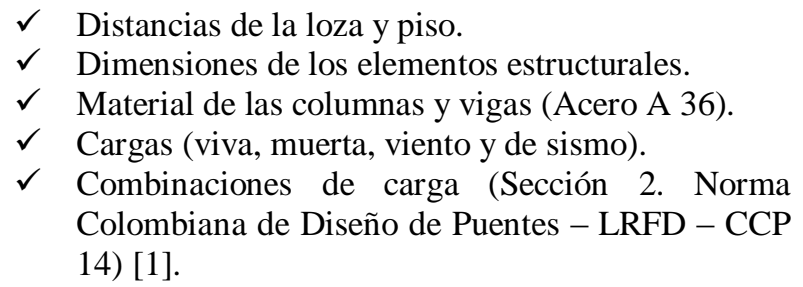

B. Distancia de la loza y piso.

Por medio del resultado de la ecuación de ancho efectivo [4] se determinó un ancho, para el nuevo modelo, de $3 \mathrm{~m}$ para que así cumpla con el ancho efectivo y la distancia del suelo a la placa se tomó de $4.5 \mathrm{~m}$ que es la distancia mínima que se pide para los puentes en zona urbana [1].

C. Dimensiones de los elementos estructurales.

$14^{\text {th }}$ LACCEI International Multi-Conference for Engineering, Education, and Technology: "Engineering Innovations for Global Sustainability”, 20-22 July 2016, San José, Costa Rica. 
Para que la estructura cumpliera con un buen servicio se tomaron las siguientes dimensiones:

TABLA V.

PERFILES UTILIZADOS PARA LA MODELACIÓN DE PUENTE PEATONAL EN ACERO.

\section{PERFILES UTILIZADOS}

\begin{tabular}{|c|c|}
\hline Arco (tubular) & $\begin{array}{l}\text { Tubo de diámetro de } 0,60 \mathrm{~m} \text { con un } \\
\text { grosor de } 0,019 \mathrm{~m}\end{array}$ \\
\hline $\begin{array}{l}\text { Pendones } \\
\text { (tubulares) }\end{array}$ & $\begin{array}{l}\text { Tubo de diámetro de } 0,09 \mathrm{~m} \text { con un } \\
\text { grosor de } 0,006 \mathrm{~m}\end{array}$ \\
\hline $\begin{array}{l}\text { Riostras } \\
\text { (tubulares) }\end{array}$ & $\begin{array}{l}\text { Tubo de diámetro de } 0,07 \mathrm{~m} \text { con un grosor } \\
\text { de } 0,006 \mathrm{~m}\end{array}$ \\
\hline $\begin{array}{l}\text { Viguetas } \\
\text { (tubulares) }\end{array}$ & $\begin{array}{l}\text { Sección rectangular de alto de } 0,12 \mathrm{~m} \text { de } \\
\text { ancho } 0,06 \mathrm{~m} \text { y un grosor de } 0,01 \mathrm{~m}\end{array}$ \\
\hline $\begin{array}{l}\text { Vigas (sección } \\
\text { comercial) }\end{array}$ & Sección europea IPE 400 \\
\hline $\begin{array}{l}\text { Riostras Columnas } \\
\text { (tubulares) }\end{array}$ & $\begin{array}{l}\text { Sección cuadrada de } 0,20 \mathrm{~m} \text { con un grosor } \\
\text { de } 0,025\end{array}$ \\
\hline $\begin{array}{c}\text { Columnas } \\
\text { (tubulares) }\end{array}$ & $\begin{array}{c}\text { Sección cuadrada de } 0,36 \mathrm{~m} \text { con un grosor } \\
\text { de } 0,025 \mathrm{~m}\end{array}$ \\
\hline
\end{tabular}

D. Cargas.

Para la modelación se utilizaron las siguientes cargas:

1) Avaluó de cargas.

A continuación se presenta el avalúo de cargas que corresponde a los esfuerzos de carga viva y muerta a los que va a estar sometida la estructura, dichos esfuerzos se calcularon con base a los parámetros que se encuentran en la Norma Colombiana de Norma Colombiana de Diseño de Puentes - LRFD - CCP 14 (Tabla VI) [1] :

TABLA VI. AVALUÓ DE CARGAS.

\begin{tabular}{|c|c|c|c|c|}
\hline \multicolumn{5}{|c|}{ AVALUO DE CARGAS } \\
\hline & & & & \\
\hline peso placa & 2,4 & $\mathrm{KN} / \mathbf{m}^{\wedge} \mathbf{2}$ & 0,240 & Ton/m^2 \\
\hline Steel Deck & 8,55 & $\mathrm{Kg} / \mathrm{m}$ & 0,009 & Ton/m \\
\hline \multirow[t]{2}{*}{ BARANDAS } & & & 0,150 & Ton/m^2 \\
\hline & & WD & 0,249 & Ton/m^2 \\
\hline \multirow[t]{2}{*}{ CARGA VIVA } & 500 & $\mathrm{Kg} / \mathrm{m}^{\wedge} \mathbf{2}$ & 0,5 & Ton/^2 \\
\hline & & WL & 0,5 & Ton/m^2 \\
\hline
\end{tabular}

2) Análisis dinámico.

La corrección realizada por análisis dinámico se evidencia en la tabla VII corroborando que las componentes en las direcciones $\mathrm{X}, \mathrm{Y}$ del sismo no distan entre sí y corresponden en magnitud al cortante basal, cabe aclarar que según normativa [1], el cortante basal equivale al $80 \%$ de la fuerza horizontal equivalente con el objeto de no llevar al límite la fuerza horizontal aplicada en estructuras regulares en planta $\mathrm{y}$ altura.

TABLA VII. VERIFICACIÓN DINÁMICA

\begin{tabular}{lccc}
\multicolumn{1}{c}{ OutputCase } & GlobalFX & GlobalFY & GlobalFZ \\
\hline Text & \multicolumn{1}{c}{ Tonf } & Tonf & \multicolumn{1}{c}{ Tonf } \\
\hline DEAD & $2,251 \mathrm{E}-13$ & $-5,6 \mathrm{E}-12$ & 40,5654 \\
\hline SISMOX & 17,5238 & 7,5074 & 0,0273 \\
\hline SISMOY & 3,7528 & 17,5236 & 0,0106 \\
\hline
\end{tabular}

El ingreso de las cargas sísmicas se realizó usando el método de análisis dinámico por medio del espectro sísmico de Bogotá establecido por la Norma Colombiana de Diseño de Puentes - LRFD - CCP 14[1].

3) Carga de viento.

En primera instancia se debe calcular VDZ (Velocidad del viento de diseño a la elevación, $\mathrm{Z}(\mathrm{km} / \mathrm{h}))$ de acuerdo a la metodología que presenta la Norma Colombiana de Diseño de Puentes - LRFD - CCP 14 [1]:

$$
V D Z=2,5 V o\left(\frac{V 10}{V B}\right) \ln \left(\frac{Z}{Z o}\right)
$$

Ecuación 3. De la Norma Colombiana de Diseño de Puentes - LRFD - CCP 14 [1].

- $\quad$ VDZ = Velocidad del viento de diseño a la elevación, $Z(\mathrm{~km} / \mathrm{h})$.

- $\quad Z=$ Altura de la estructura a la cual se calculan las cargas de viento medida desde el nivel del terreno, o desde el nivel del agua > a $10000 \mathrm{~mm}$.

- $\mathrm{Zo}=$ Se elige de la Tabla del código colombiano de puentes 3.8.1.1-1.

- Vo= Se elige de la Tabla del código colombiano de puentes 3.8.1.1-1.

- $\mathrm{VB}=$ Velocidad básica del viento a $160 \mathrm{Km} / \mathrm{h}$ a $10000 \mathrm{~mm}$ de altura.

- $\mathrm{V} 30=$ Velocidad del viento a $10000 \mathrm{~mm}$ sobre el nivel del terreno $(\mathrm{Km} / \mathrm{h})$.

Elección de Vo, Zo, V10, VB De acuerdo a la Norma Colombiana de Diseño de Puentes - LRFD - CCP 14 [1]:

- $\mathrm{Vo}=19,30 \mathrm{Km} / \mathrm{h}$ para ciudad.

- $\mathrm{Zo}=2,50 \mathrm{~m}$ para ciudad.

- $\mathrm{V} 10=\mathrm{VB}=160 \mathrm{Km} / \mathrm{h}$ por norma de la tabla 3.8.1.1-1

$14^{\text {th }}$ LACCEI International Multi-Conference for Engineering, Education, and Technology: "Engineering Innovations for Global Sustainability”, 20-22 July 2016, San José, Costa Rica. 


$$
P D=P B\left(\frac{V D Z^{2}}{V B^{2}}\right)=P B\left(\frac{V D Z^{2}}{25600}\right)
$$

Ecuación 4. 3.8.1.2.1-1 De la NORMA COLOMBIANA

DE DISEÑO DE PUENTES - LRFD - CCP 14 [1].

- $\quad \mathrm{PB}=$ Presión del viento básica especificada en la tabla 3.8.1.2.1-1 (Mpa) de la Norma Colombiana de Diseño de Puentes - LRFD - CCP 14

- $\mathrm{Z}=$ Altura del arco en metros

Tabla VIII. Selección de PB para Sotavento para Cerchas, Columnas y Arcos [1].

Tabla 3.8.1.2.1-1 - Presiones Básicas, $P_{B}$, Correspondientes a $V_{B}=160 \mathrm{~km} / \mathrm{h}$

\begin{tabular}{|c|c|c|}
\hline $\begin{array}{c}\text { Componente de la } \\
\text { Subestructura }\end{array}$ & $\begin{array}{c}\text { Carga de } \\
\text { Barlovento } \\
\mathrm{MPa}\end{array}$ & $\begin{array}{c}\text { Carga de } \\
\text { Sotavento } \\
\mathrm{MPa}\end{array}$ \\
\hline $\begin{array}{c}\text { Cerchas, Columnas, } \\
\text { y Arcos }\end{array}$ & 0.0024 & 0.0012 \\
\hline Vigas & 0.0024 & $\mathrm{NA}$ \\
\hline $\begin{array}{c}\text { Superficies grandes } \\
\text { Planas }\end{array}$ & 0.0019 & $\mathrm{NA}$ \\
\hline
\end{tabular}

De acuerdo a la tabla VIII y basados en la geometría del modelo se elige una carga de presiones básicas PB de 0,0012 Mpa suponiendo una dirección del viento de Sotavento.

$V D Z=2,5 * 19,30 \mathrm{Km} /$

$h\left(\frac{160 \mathrm{Km} / \mathrm{h}}{160 \mathrm{Km} / \mathrm{h}}\right) \operatorname{Ln}\left(\frac{11,5}{2,5}\right)=73,63 \mathrm{Km} / \mathrm{h}$

$\mathrm{PD}=\quad 0, \quad 0012(\llbracket 73,63 \mathrm{Km} / \mathrm{h} \rrbracket \wedge 2 / \llbracket 160 \mathrm{Km} / \mathrm{h} \rrbracket \wedge 2)$ $=0,000254 \mathrm{Mpa}$

- $\quad \mathrm{PD}=$ Presión de viento de diseño.

E. Combinaciones de carga.

Las combinaciones de carga que se usaron son las especificadas en la Norma Colombiana de Diseño de Puentes - LRFD - CCP 14 para el cálculo de derivas en elementos estructurales [1].

TABLA IX

TIPOS DE CARGA QUE AFECTAN EL PUENTE PROPUESTO.

\begin{tabular}{cc}
\hline TIPO DE CRAGA & NOMENCLATURA \\
\hline Carga muerta & DC \\
\hline Carga viva peatonal & PL \\
\hline Viento dirección $\mathbf{x}$ & WSx \\
\hline Viento dirección $\mathbf{y}$ & WSy \\
\hline Sismo dirección $\mathbf{x}$ & EQx \\
\hline Sismo dirección $\mathbf{y}$ & EQy \\
\hline
\end{tabular}

TABLA X. COMBINACIONES DE CARGA

\begin{tabular}{|c|c|c|c|c|c|c|}
\hline \multirow{2}{*}{$\begin{array}{c}\text { Estado límite de } \\
\text { la combinación } \\
\text { de carga }\end{array}$} & \multicolumn{6}{|c|}{ TIPOS DE CARGA } \\
\hline & DC & PL & WSx & WSy & EQx & EQy \\
\hline Resistencia I & 1,25 & 1,75 & - & - & - & - \\
\hline Resistencia ll & 1,25 & 1,35 & - & - & - & - \\
\hline \multirow{4}{*}{ Resistencia Ill } & 1,25 & - & 1,40 & - & - & - \\
\hline & 1,25 & - & $-1,40$ & - & - & - \\
\hline & 1,25 & - & - & 1,40 & - & - \\
\hline & 1,25 & - & - & $-1,40$ & - & - \\
\hline Resistencia IV & 1,25 & - & - & - & - & - \\
\hline \multirow{4}{*}{ Resistencia V } & 1,25 & 1,35 & 0,40 & - & - & - \\
\hline & 1,25 & 1,35 & $-0,40$ & - & - & - \\
\hline & 1,25 & 1,35 & - & 0,40 & - & - \\
\hline & 1,25 & 1,35 & - & $-0,40$ & - & - \\
\hline \multirow{2}{*}{$\begin{array}{c}\text { Evento Extremo } \\
1\end{array}$} & 1,25 & 1,00 & - & - & 1,00 & - \\
\hline & 1,25 & 1,00 & - & - & - & 1,00 \\
\hline $\begin{array}{c}\text { Evento Extremo } \\
\text { Il }\end{array}$ & 1,25 & 0,50 & - & - & - & - \\
\hline
\end{tabular}

\section{RESULTADOS DE LA MODELACIÓN.}

Luego de haber insertado todos los datos necesarios para hacer el análisis estructural del modelo de puente peatonal propuesto, se procede a hacer el análisis dinámico (Derivas, no deben exceder el $1 \%$ de la altura de la estructura la cual es de 11,5 metros) y el diseño de los elementos estructurales que conforman la estructura como se puede ver en la Ilustración 6 y 7 respectivamente:

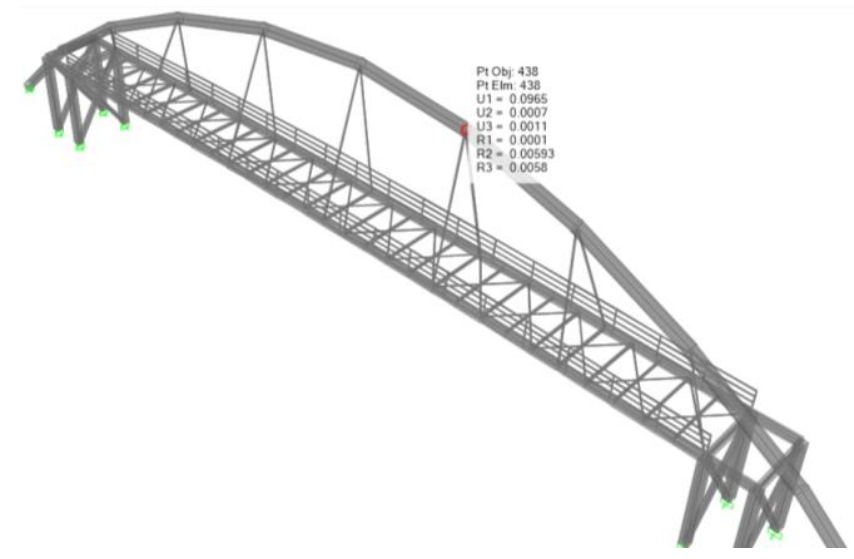

Ilustración 6.Comprobación de Derivas.

$14^{\text {th }}$ LACCEI International Multi-Conference for Engineering, Education, and Technology: "Engineering Innovations for Global Sustainability", 20-22 July 2016, San José, Costa Rica. 


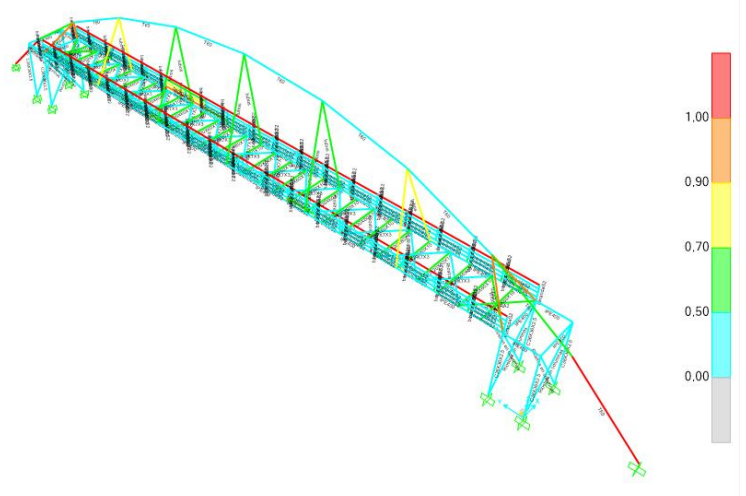

Ilustración 7.Chequeo de diseños estructurales del modelo propuesto.

De acuerdo a la Ilustración 6, se evidencia que el parámetro de derivas cumple de acuerdo a las normativas vigentes las cuales documentan, que las derivas deben corresponder al $1 \%$ de la altura de la estructura [1]. En esta investigación se permite una deriva máxima de 0,115 y la deriva obtenida al analizar el modelo en el punto más crítico fue del orden de 0,097 como se observa en la tabla XI. Comparando estos dos valores se puede concluir que la estructura no sobrepasa la deriva máxima permitida por la norma y se puede decir que tiene una rigidez estable.

En la Ilustración 7, se logra evidenciar que los perfiles estructurales elegidos presentan un esfuerzo máximo que varía de 0 a $80 \%$ (ver escala Ilustración 7), dejando así un $20 \%$ de confiabilidad. Desde el punto de vista ingenieril, este es un parámetro muy importante debido a que permite un margen amplio en caso de momentos extremos inesperados tales como sismos, explosivos que puedan afectar la estructura. Se busca con esto que la estructura logre responder a fuerzas inesperadas con el fin de salvaguardad a la población en caso de dichos eventos.

TABLA XI.

DESPLAZAMIENTOS MÁXIMOS DE LA ESTRUCTURA

\begin{tabular}{|c|c|c|c|c|c|c|}
\hline \multicolumn{7}{|c|}{ TABLE: Joint Displacements } \\
\hline $\begin{array}{c}\text { Join } \\
\mathbf{t}\end{array}$ & $\begin{array}{c}\text { OutputCa } \\
\text { se } \\
\end{array}$ & CaseType & $\begin{array}{c}\text { StepTyp } \\
\text { e } \\
\end{array}$ & U1 & $\mathbf{U} 2$ & U3 \\
\hline Text & Text & Text & Text & $\mathrm{m}$ & $\mathrm{m}$ & $\mathrm{m}$ \\
\hline 439 & SISMOX & $\begin{array}{l}\text { LinRespSp } \\
\text { ec }\end{array}$ & Max & 0,0963 & 0,00021 & 0,0000 \\
\hline 438 & SISMOX & $\begin{array}{l}\text { LinRespSp } \\
\text { ec }\end{array}$ & Max & 0,0956 & 0,00024 & 0,0003 \\
\hline 440 & SISMOX & $\begin{array}{l}\text { LinRespSp } \\
\text { ec }\end{array}$ & Max & 0,0856 & 0,00024 & 0,0003 \\
\hline 141 & SISMO & LinRespSp & & 0,0973 & 0,00024 & 0,0003 \\
\hline
\end{tabular}

\section{CONCLUSIONES.}

De acuerdo al desarrollo de cada una de las fases que plantean las siguientes conclusiones:
Se propuso posibles mejoras en la movilidad peatonal del puente peatonal ubicado en la Avenida 68 entre las calles 63 y 64 , mediante modelación de un nuevo puente peatonal en estructura metálica debido a que el puente existente no cumple con los parámetros requeridos para su uso como se evidencia en los resultados de ancho libre en la ecuación 2 [4] por lo tanto se modelo un nuevo puente que cumple con los parámetros establecidos por la Norma Colombiana de Diseño de Puentes - LRFD - CCP 14 en la Ilustración 6, se evidencia que el parámetro de derivas cumple de acuerdo a las normativas vigentes las cuales documentan, que las derivas deben corresponder al $1 \%$ de la altura de la estructura [1]. En esta investigación se obtiene una deriva máxima de 0,115 y la deriva obtenida al analizar el modelo en el punto más crítico fue del orden de 0,097 como se observa en la tabla XI. Comparando estos dos valores se puede concluir que la estructura no sobrepasa la deriva máxima permitida por la norma y se puede decir que tiene una rigidez estable. Se consideraron las cargas actuantes en la estructura, las posibles combinaciones de carga que se puedan presentar (Tabla X), Análisis dinámico teniendo en cuenta la zona de ubicación del puente peatonal con el fin de simular las condiciones de sismo que puedan presentarse para que la modelación sea lo más realista posible.

Por otra parte, desde el punto de vista geométrico se mejoró las condiciones que presenta el puente peatonal actual frente al servicio, debido a que, se eligieron secciones más amplias en el tablero de acuerdo a los aforos presentados (Tabla II, III, IV) que por la movilidad del sector es un parámetro de importancia.

- Se identificó las causas por las cuales el puente peatonal ubicado en la Avenida 68 entre calles 63 y 64 se ha deteriorado a lo largo del tiempo, debido a que se evidencio fracturas en elementos estructurales tales como columnas, vigas y tablero (Ilustraciones 2-5), que pueden generar a futuro un colapso y generar pérdidas de vidas humanas.

- Se describió detalladamente la geometría del puente peatonal de la Avenida 68 entre calles 63 y 64 por medio de mediciones con cinta métrica, se logró evidenciar secciones (losa) que no satisfacen las necesidades de la población ya que esta corresponde a una zona de alta movilidad peatonal debido a la cercanía de centros recreativos y educativos.

- Se modeló la alternativa más adecuada de un puente peatonal en acero con especificaciones de la norma con el objeto de satisfacer la demanda del sector, soporte y la resistencia que causan las cargas aplicadas a la estructura como lo son la carga muerta, carga viva, las cargas de sismo y las cargas de viento. También con estas cargas se buscó que el puente cumpliera con los desplazamientos que permite la norma que es de un máximo del $1 \%$ de la altura de la estructura pero sin que los elementos estén trabajando por encima del $100 \%$ de su capacidad [1].

$14^{\text {th }}$ LACCEI International Multi-Conference for Engineering, Education, and Technology: "Engineering Innovations for Global Sustainability", 20-22 July 2016, San José, Costa Rica. 


\section{RECOMENDACIONES.}

- Teniendo en cuenta que esta investigación se enfocó en el modelo y diseño estructural del puente peatonal, se recomienda ampliar el estudio desde la parte del suelo para complementar los resultados y así proponer una mejor alternativa.

- Se recomienda también que si el puente es construido se le debe aplicar una pintura anticorrosiva para que soporte los tipos de clima que se presentan en la ciudad de Bogotá.

- Se recomienda realizar aforos en los días que hallan eventos en las zonas públicas debido a que se puede duplicar o triplicar el número de peatones que posiblemente utilizaran el puente.

\section{REFERENCIAS.}

[1]Norma Colombiana de Diseño de Puentes - LRFD - CCP 14. Convenio de asociación No 1314 de 2013. Bogotá: Ministerio de transporte, Instituto nacional de vías (INVIAS) en convenio con la Asociación Colombiana de Ingeniería Sísmica (AIS), 2013, p.1503.

[2]Instituto de desarrollo urbano [en línea]. Disponible en: <http://www.idu.gov.co/web/guest/puentes_peatonales [citado en 11 de abril de 2015].

[3]Instituto de desarrollo URBANO (IDU). Criterios y parámetros de diseño para los puentes peatonales [en línea]. (2007). Disponible en: < http://webidu.idu.gov.co:9090/jspui/bitstream/123456789/2951 1/6/60015554-05.PDF > 8 p. [Citado en 1 de noviembre de 2015].

[4]TORRES, Julio Oswaldo. Estudio de tránsito construcción del puente peatonal "Nazareth-Rios Blanca" ubicado en la Localidad de Sumapaz [en línea]. (2011). 44 p. [Citado en 1 de noviembre de 2015].

$14^{\text {th }}$ LACCEI International Multi-Conference for Engineering, Education, and Technology: "Engineering Innovations for Global Sustainability", 20-22 July 2016, San José, Costa Rica. 
14 ${ }^{\text {th }}$ LACCEI International Multi-Conference for Engineering, Education, and Technology: "Engineering Innovations for Global Sustainability", 20-22 July 2016, San José, Costa Rica. 